\title{
Local Heat Transfer to an Evaporating Superhydrophobic Droplet
}

\author{
M.J. Gibbons ${ }^{\mathrm{a}, *}$, P. Di Marco ${ }^{\mathrm{b}}$, A.J. Robinson ${ }^{\mathrm{a}}$ \\ ${ }^{a}$ Department of Mechanical, Manufacturing and Biomedical Engineering, Trinity College Dublin, the University of Dublin, Dublin 2, \\ Ireland. \\ ${ }^{b}$ DESTEC, University of Pisa, Italy.
}

\begin{abstract}
The heat flux distribution beneath a superhydrophobic evaporating droplet has been explored. High speed, high resolution infrared thermography is employed to measure the heat transfer characteristic of the evaporating droplet. Optical imaging and analytical techniques are used the capture droplet dynamics over the course of its evaporation. The droplet evaporated with a receding contact line predominantly in the CCA regime. The peak local convective heat transfer was located at the triple contact line over the entire evaporation period. Peak and average heat fluxes were shown to increase as the evaporation proceeded due to the increasing contact line length density and liquid-gas interface temperature. The total thermal power across the solid-liquid interface decreased due to the decreasing solid-liquid surface area. The average heat flux to the evaporating droplet was shown to vary linearly with contact line length density.
\end{abstract}

Keywords: Droplet, Evaporation, Heat transfer, Contact line, Superhydrophobic

\section{Introduction}

Evaporation of sessile drops is of significant scientific and engineering interest. It represents a common natural phenomena that is not fully understood while at the same time is used in many industrial fields, ranging from DNA mapping to evaporative spray cooling [1-13].

Droplet evaporation dynamics depends on many factors, including though not limited to wettability, the evaporation flux at the interface and the triple line, the substrate temperature, external fields and thermocapillarity, and these have been researched extensively over the past decade [12, 14-28]. From a thermal transport standpoint, the process is a complex interaction of diffusion within the substrate, buoyant convection in the gas and liquid phases, contact line evaporation, vapour diffusion, evaporative cooling at the liquid-gas interface and possible Marangoni effects [12, 14-29]. The droplet evaporation process is clearly very complex and even though considerable progress has been made with regard to understanding the thermal and fluid transport processes, they are still not fully understood, in particular with regard to the conjugate heat transfer near the triple contact line [29].

The contact line or triple line is defined as the region where the gas, liquid and solid phases intersect. Droplet evaporation can occur in four primary modes: constant contact angle (CCA), constant contact radius (CCR), a mixed mode, and a stick slip mode. Both CCA and CCR were first introduced by Picknett and Bexon [14]. In CCA mode, the droplet's contact angle remains fixed with a

\footnotetext{
* Corresponding author

Email address: Michael.Gibbons@tcd.ie (M.J. Gibbons)
}

decreasing base radius $r_{b}$, whereas for CCR mode, the droplet's radius is fixed (pinned) and droplet evaporation occurs with a decreasing contact angle. In mixed mode evaporation both the contact angle and radius decreases as the evaporation proceeds [30]. Finally in the stick-slip mode the contact line oscillates between pinning and unpinning [31]. Which modes occur is dependent on the droplet and substrate properties [24].

Conjugate heat transfer near the contact line during droplet evaporation on heated substrates is a topic that still requires significant research. For hydrophilic droplets, it is known that the adsorbed layer forms as a result of the strong adhesion forces between the liquid and the solid. As depicted in Fig. 1a, the result of these strong adhesion forces creates a contact line region that has multiple length scales, ranging from nanometer at the adsorbed film region to millimeter in the bulk droplet. The adsorbed film region is characterised by long range intermolecular forces. This disjoining pressure result in a flat liquid-vapour interface of a few nanometers thick (10 $20 \mathrm{~nm}$ ) [32] and prevents evaporation occurring in this region [33]. The transition region is defined by growing film thickness which results in a reduction in the long range intermolecular forces. This region experience the highest heat fluxes across the droplet as a result of the low thermal resistance from the small film thickness $(1-3 \mu \mathrm{m})$ [32]. The total length of the adsorbed film and transition region varies from $0.5 \mu \mathrm{m}$ to $10-20 \mu \mathrm{m}$ [32]. As the film thickness increases from the transition region into the intrinsic meniscus and micro-convection regions, so too does the thermal resistance resulting in a decrease in the local heat flux. Both the intrinsic meniscus and micro-convection 


\begin{tabular}{|c|c|c|c|}
\hline \multicolumn{4}{|c|}{ Nomenclature } \\
\hline$A$ & Area $\left[\mathrm{mm}^{2}\right]$ & $\infty$ & Bulk or infinity \\
\hline$C$ & Curvature $\left[\mathrm{mm}^{-1}\right]$ & $a g$ & Air gap \\
\hline$C_{p}$ & Specific heat capacity $[\mathrm{J}]$ & $b$ & base \\
\hline$E$ & Energy $\left[\mathrm{W} \mathrm{m}{ }^{-1} \mathrm{~K}^{-1}\right]$ & cap & Capacitive or storage \\
\hline$H$ & height [mm] & cond & Conduction \\
\hline$I$ & Current $[\mathrm{A}]$ & $d$ & Droplet \\
\hline$k$ & Thermal conductivity $\left.\left[\mathrm{W} \mathrm{m}^{-1} \mathrm{~K}^{-1}\right]\right]$ & $f$ & Foil \\
\hline$q^{\prime \prime}$ & Heat flux $\left[\mathrm{W} \mathrm{m}^{-2}\right]$ & gen & Generated \\
\hline$R$ & Thermal resistance $\left[\mathrm{K} \mathrm{W}^{-1}\right]$ & $l c$ & Lateral conduction \\
\hline$r$ & Radius $[\mathrm{mm}]$ & $\lg$ & liquid-gas \\
\hline$S$ & Coordinate $[\mathrm{mm}]$ & $p$ & Paint \\
\hline$T$ & Temperature $[\mathrm{K}]$ & $\mathrm{rad}$ & Radiation \\
\hline$t$ & Time $[\mathrm{min}]$ & $s$ & Heated substrate \\
\hline \multirow[t]{2}{*}{$V$} & Voltage $[V]$ & $s l$ & solid-liquid \\
\hline & & $T$ & apex \\
\hline \multicolumn{2}{|c|}{ Dimensionless Numbers } & $w f$ & Working fluid \\
\hline$B i$ & $B i=h L / k[-]$ & $x, y, z$ & Coordinate direction \\
\hline \multicolumn{2}{|c|}{ Greek Symbols } & \multicolumn{2}{|c|}{ Acronyms } \\
\hline$\delta$ & Thickness $[\mu \mathrm{m}]$ & $\mathrm{CAH}$ & Contact angle hysteresis \\
\hline$\gamma$ & Surface tension $\left.\left[\mathrm{N} \mathrm{m}^{-1}\right]\right]$ & $\mathrm{CCA}$ & Constant contact angle \\
\hline$\rho$ & Density $\left[\mathrm{kg} \mathrm{m}^{-3}\right]$ & CCR & Constant contact radius \\
\hline$\sigma$ & Stefan-Boltzmann constant $\left[\mathrm{W} \mathrm{m}^{-2} \mathrm{~K}^{-4}\right]$ & CLL & Contact line length density \\
\hline$\theta$ & Contact angle $\left[^{\circ}\right]$ & $\mathrm{DC}$ & Direct current \\
\hline \multirow{2}{*}{$\varepsilon$} & Emissivity [-] & IR & Infrared \\
\hline & & PEEK & Polyether ether ketone \\
\hline$S u b$ & & PU & Percentage uncertainty \\
\hline
\end{tabular}

regions are characterised by surface tension and inertial forces [21]. The net thermal boundary resistance $R_{n e t}$ is the sum of the solid-liquid $R_{s l}$ and the liquid-gas $R_{l g}$ thermal resistance, with $R_{l g}$ typically 10 - 100 times greater in magnitude than $R_{s l}$ [24]. If the evaporating vapour is lighter than the surround medium (air) convection may occur [23]. Vapour concentration gradients at the liquid-gas interface establishes natural convection currents in the surrounding gas. These currents influence the advection and diffusion of the vapour [25, 26]. If an air draft over the droplet is present during evaporation, then advection will dominate at a given distance from the droplet surface [23].

It is well established that the low thermal resistance of the liquid in the transition region results in proportionately high evaporative heat and mass flux. From a heat transfer point of view, this has been observed as a 'coffeering' shaped heat flux distribution beneath a hydrophilic droplet on heated substrates [32]. Thus, it can be said that although there is significant work to be done to fully understand the conjugate heat transfer phenomenon for hydrophilic droplets, the underlying mechanics of the phenomenon is known. For non-wetting droplets, the droplet mechanics are significantly different since the adhesion forces between the solid and liquid are weak. In particular, for superhydrophobic droplets it is predicted that there is a greatly reduced adsorbed film region [12] and this mitigates the formation of a micrometer-scale transition region, and this shown diagrammatically in Fig. 1b. Thus, the conjugate heat transfer mechanisms are quite different compared with the hydrophilic case. To the authors' knowledge, the only study that has investigated the conjugate heat transfer mechanisms at the contact line of hydrophobic and superhydrophobic droplets is the numerical investigation by Pan et al. [34]. Considering free evaporation, a very important result from this study was that when evaporative cooling of the gas-liquid interface was included, it establishes a temperature gradient along the droplet interface, with the highest temperature occurring near the contact line due to the proximity with the hotter, though not heated, surface. This established the highest evaporation flux due to the increase in the local saturation pressure which induces a high vapour fraction gradient which drives vapour diffusion and overcomes the geometric confinement in this region, which tends to impede diffusion. It is thus concluded that for free evaporation, contact line evaporation is also highest at the contact line though 
(a)

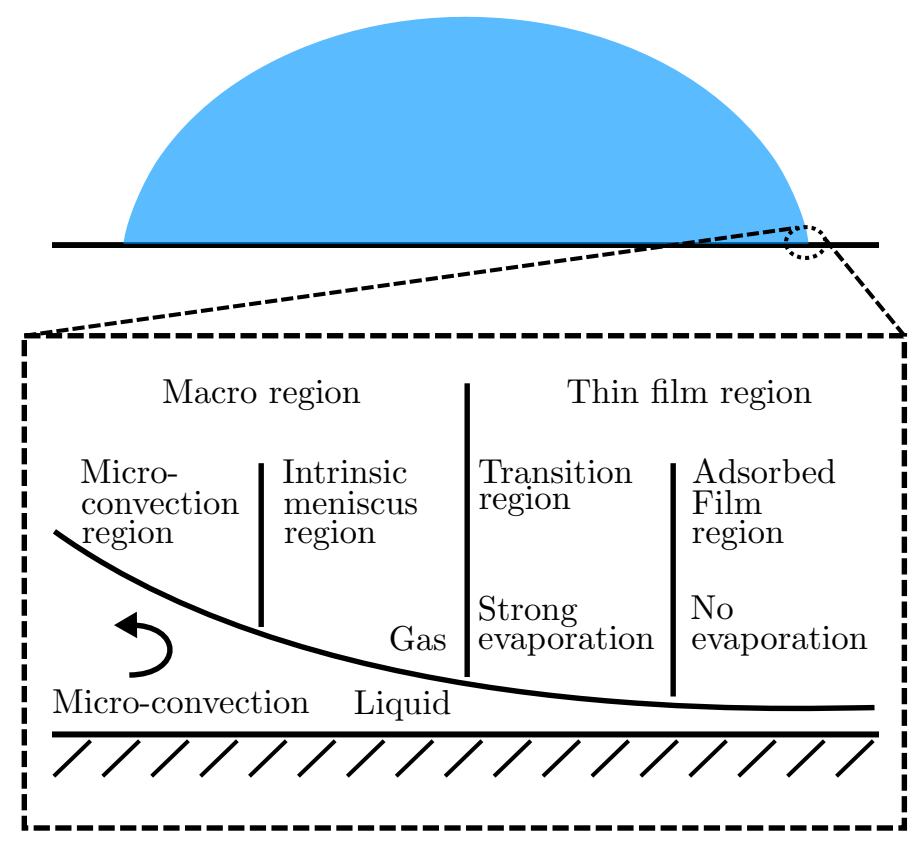

(b)

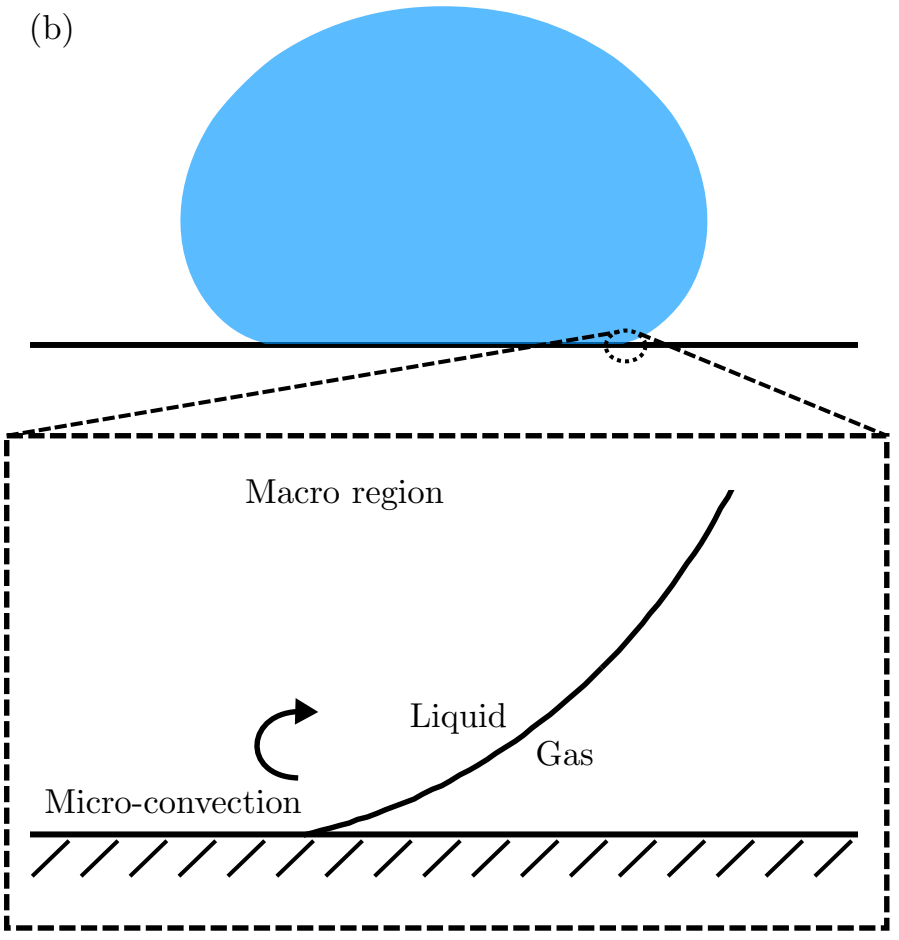

Figure 1: Droplet evaporation at the contact line. (a) hydrophilic droplet [21] and (b) hydrophobic droplet.

due to quite different mechanisms compared with wetting droplets. The open question now is whether this mechanism translates into a notably high local surface heat flux for non-wetting droplets on heated substrates.

This investigation aims to contribute to the understanding of droplet evaporation by measuring the heat flux beneath an evaporating superhydrophobic droplet through a full evaporation cycle. To the best of our knowledge, this represents the first study to provide the spatial distribution of the surface heat flux beneath a non-wetting evaporating droplet as well as the first to consider time varying heat flux distributions for a full evaporation event. Simultaneously, the droplet geometry is considered so as to gain some insight into the droplet mechanics and the heat transfer. For this particular study a partial non-wetting water droplet is considered from an initial volume of $80 \mu \mathrm{L}$ until it fully evaporates.

\section{Experimental Apparatus and Data Reduction}

The experimental apparatus consists of two primary components; the heat transfer section and the imaging system. These are illustrated in the rig schematic in Fig. 2.

\subsection{Heat transfer section}

The thermal exchange section (shown in Fig. 3) consists of a $25 \mu \mathrm{m}$ thick, $140 \times 80 \mathrm{~mm}^{2}$ Stainless Steel 316 foil (Goodfellow, Fe/Cr18/Ni10/Mo3, P/N: 505-400-04). The foil is clamped between two copper bus bars, which are electrically connected to a Lambda GENESYS 6-200 DC power supply (P/N: GEN 6-200). The copper bus bars are mounted to a polyether ether ketone (PEEK, Ketron 1000 PEEK) housing. A tensioning system is employed in order to counteract foil warping at higher foil temperature gradients. One set of bus bars are rigidly fixed to the PEEK housing while the other is spring loaded. The $25 \mu \mathrm{m}$ foil is stretched across a $140 \times 100 \times 13 \mathrm{~mm}^{3}$ central PEEK piece.

The voltage drop and current through the heated substrate was measured using two Hewlett-Packard 34401A digital multimeters (DMM). The voltage reading is taken across the steel foil between the copper busbars. The DMMs are connected in series via a GPIB serial connec-

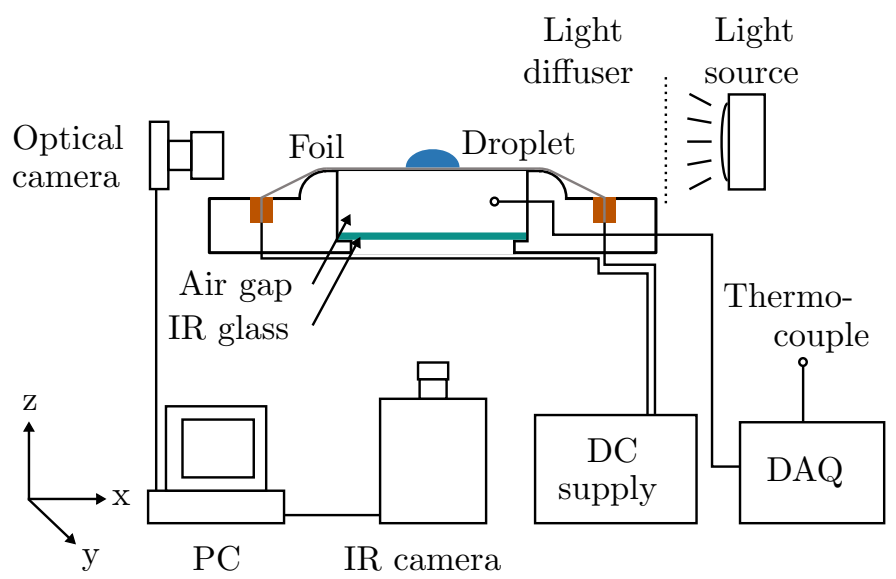

Figure 2: Schematic diagram of experimental set-up. 


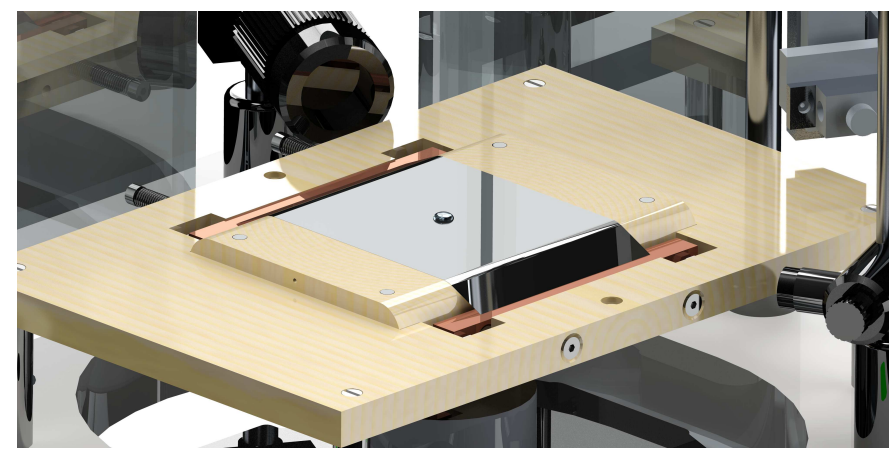

Figure 3: Droplet evaporation test facility.

tion and National instrument (NI) USB - GPIB cable to the dedicated computer. The current was scaled into an easily measurable voltage drop using a Murata 50 A 50 $\mathrm{mV} \pm 0.25 \%$ shunt $(\mathrm{P} / \mathrm{N}: 3020-01096-0)$.

The top side of the steel foil was superhydrophobically coated using Glaco Mirror Coat Zero. It was applied in a manner similar to that of Malavasi et al. [35]. The superhydrophobic coating of the heated interface results in a pretesting ambient proceeding contact angle of $\sim 160^{\circ}$ with $1^{\circ}$ contact angle hysteresis $(\mathrm{CAH})$. This contact angle and low $\mathrm{CAH}$ corresponds to the superhydrophic case as previously defined by Drelich and Chibowski [36]. The central PEEK piece serves to house a $70 \times 70 \times 2 \mathrm{~mm}^{3}$ infrared (IR) transparent Calcium Fluoride $\left(\mathrm{CaF}_{2}\right)$ window. The top of this IR window is positioned $7 \mathrm{~mm}$ below the underside of the foil, establishing an air cavity that acts as a thermal barrier. A $1.6 \mathrm{~mm}$ exposed T-type thermocouple is used to measure the air temperature at the midpoint of the air gap cavity. The underside of the foil is exposed for direct temperature measurement by an infrared camera. It is coated with a thin layer of matt black paint of known emissivity to facilitate accurate temperature measurement. The heat transfer section is mounted on a acetal baseplate (Fig. $4 \mathrm{a}$ ), $400 \times 320 \times 30 \mathrm{~mm}^{3}$, and is supported on a $40 \times 40 \mathrm{~mm}^{2}$ aluminium profile frame (P/N: 3842993120/3000).

Table 1: Foil and paint properties.

\begin{tabular}{lrl}
\hline Foil thickness, $\delta_{f}$ & 25 & $\mu \mathrm{m}$ \\
Density, $\rho_{f}$ & 7960 & $\mathrm{~kg} \mathrm{~m}^{-3}$ \\
Thermal conductivity, $k_{f}$ & 16.3 & $\mathrm{~W} \mathrm{~m}^{-1} \mathrm{~K}^{-1}$ \\
Specific heat, $\mathrm{C}_{p, f}$ & 502 & $\mathrm{~J} \mathrm{~kg}^{-1} \mathrm{~K}^{-1}$ \\
${\text { Surface Roughness, } R a_{f}}_{\mathrm{CaF}_{2} \text { Emissivity, } \varepsilon_{C a F_{2}}}^{176}$ & $\mathrm{~nm}$ \\
${\text { Paint layer thickness, } \delta_{p}}^{\text {Density, } \rho_{p}}$ & 0.2 & - \\
Thermal conductivity, $k_{p}$ & 10.52 & $\mu \mathrm{m}$ \\
Specific heat, C,$p$ & 1261 & $\mathrm{~kg} \mathrm{~m}^{-3}$ \\
Paint Emissivity, $\varepsilon_{p}$ & 0.095 & $\mathrm{~W} \mathrm{~m}^{-1} \mathrm{~K}^{-1}$ \\
\hline
\end{tabular}

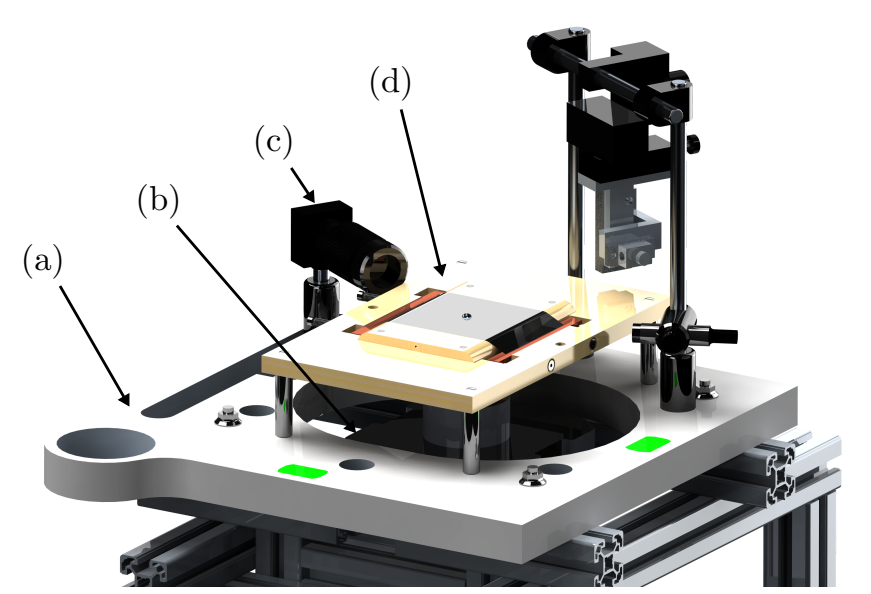

Figure 4: Thermal platform configuration. (a) acetal baseplate, (b) IR camera, (c) optical camera, and (d) thermal exchange section.

\subsubsection{Material Properties}

The foil and paint properties are outlined in Table 1. The paint thickness was determined using a Dektak $6 \mathrm{M}$ programmable surface profiler. The foil properties were defined using the supplied Goodfellows data sheet. The density of the paint was determined by coating a known volume of paint and comparing the mass before and after painting. The thermal conductivity of the paint layer was determined by means of Hot Disk Transient Plane Source Method, while the specific heat value was taken from data tabulated by Raghu and Philip [37]. The surface roughness of the coated steel foil was determined using a MicroXAM White light Interferometric Surface Profiler.

\subsection{Imaging system}

The imaging system consisted of two parts; a Chameleon CMLN-1352M optical camera and a FLIR SC6000 thermal imaging camera. Both cameras are controlled by a dedicated computer. The optical camera is mounted to the acetal baseplace as shown in Fig 4b, and was focused on the evaporating droplet during testing. The camera was fitted with a long distance microscopic lens (Infinity Proximity InfiniMite Gamma lens with a DL Tube $/ 2 \times, \mathrm{P} / \mathrm{N}$ : 57- 724 and 39-686). Captured images had a resolution of $1280 \times 960$ pixels, with a pixel width corresponding to $10.5 \mu \mathrm{m}$.

The FLIR SC6000 high resolution, high frame rate IR camera was used to capture the thermal footprint of the evaporating droplet. The camera is mounted to the aluminium profile, directly below the thermal exchange surface. The IR camera is fitted with a $25 \mathrm{~mm}$ focal length lens. The frame rate and capturing period is controlled via an external pulse generated by a NI 9401 Data Acquisition Module (DAQ) in LabVIEW. The thermal camera is set to record an image that is $400 \times 400$ pixels with each pixel corresponding to a width of $160 \mu \mathrm{m}$. 
Table 2: Data acquisition sample rate.

\begin{tabular}{|c|c|c|}
\hline IR camera & 200 & $\mathrm{~Hz}$ \\
\hline Optical camera ${ }^{1}$ & 2 & $\mathrm{H}_{z}$ \\
\hline Ambient temperature, $T_{\infty}$ & 2 & $\mathrm{H}_{2}$ \\
\hline Air gap temperature, $T_{a g}$ & 2 & $\mathrm{H}_{z}$ \\
\hline Surface voltage drop, $V_{s}$ & 20 & $\mathrm{H}_{2}$ \\
\hline Surface current, $I_{s}$ & 20 & $\mathrm{H}_{2}$ \\
\hline
\end{tabular}

\subsection{Experimental parameters}

The working fluid was deionised water from SigmaAldrich. The initial droplet volume was set at $80 \mu \mathrm{L}$. The volume was achieved using a KDS 200 dual syringe infusion pump $(\mathrm{P} / \mathrm{N}:$ 789200) and a $500 \mu \mathrm{L}$ Hamilton 1750 gastight syringe $(\mathrm{P} / \mathrm{N}$ : 81262). The syringe pump was set to the desired volume and deposited on the surface using $100 \mu \mathrm{m}$ inner diameter FEP tubing (DuPont, P/N: 147420). The heated substrate temperature was assumed to be uniform across its thickness at a constant generation heat flux of $912 \mathrm{~W} \mathrm{~m}^{-2}$. This corresponded to a surface temperature of $\sim 70^{\circ} \mathrm{C}$ in the absence of droplet evaporation at steady state conditions.

\subsection{Experimental procedure}

Experiments were conducted in ambient surroundings and atmospheric pressure.

1. The $80 \mu \mathrm{L}$ deionised water droplet was placed in the centre of the stainless steel substrate.

2. A calibration image was captured to determine pixel size for post processing.

3. The start of testing was triggered using a custom built LabVIEW routine (VI). Once the VI was started it triggers the thermal imaging camera, optical camera and the heated substrate power supply, while simultaneously acquiring thermocouple, heated substrate voltage drop and circuit current readings. Data was acquired for 1 second every 60 seconds for each sub-point. The total test time for all tests was $\sim$ $32 \mathrm{~min}$. This results in 33 sub-points per test, with each sub-point separated by 59 seconds. All droplets were completely evaporated by the completion of each test. A list of the sampling rate at each subpoint is shown in Table 2.

\subsection{Data analysis and processing}

Two sets of data analysis were conducted. The first is the local temperature field of the evaporating droplet to investigate the local heat transfer across the solid-liquid interface, and the second is the captured optical image to determine how the droplet geometric parameters vary over the evaporation period.

\footnotetext{
${ }^{1}$ Optical camera was continuously recording across entire test period.
}

\subsubsection{Thermal image processing}

The captured thermal images are processed using MATLAB. Data processing can be divided into three phases; image conversion, heat transfer analysis and averaging.

\section{Image conversion}

For each sub-point the captured thermal data is imported into MATLAB. Each frame is extracted and the bad pixel, gain, offset and counts to temperature conversion are applied. This results in $200400 \times 400$ thermal images stored in a 3D array.

\section{Heat transfer analysis}

In order to compute the thermal energy convected to the droplet, various fluid, foil and paint properties (see Table 1) are input along with the averaged values for the relevant acquired data (thermocouple, foil voltage drop and circuit current). In order to evaluate the convective heat flux to the droplet, an element by element based energy balance is applied to each pixel of the recorded thermal image. The energy balance can be defined as [38]:

$$
\dot{E}_{\text {in }}+\dot{E}_{\text {gen }}-\dot{E}_{\text {out }}=\dot{E}_{\text {st }}
$$

It is assumed that there is uniform heat generation within the foil and that the temperature across the thickness of the foil and paint layers are constant as $\mathrm{Bi} \ll 1$ for both the paint and foil layers. This results in the following expression for the convective heat flux:

$$
q_{e c}^{\prime \prime}=q_{g e n}^{\prime \prime}-q_{c o n d}^{\prime \prime}-q_{r a d, b}^{\prime \prime}-q_{l c}^{\prime \prime}-q_{c a p}^{\prime \prime}
$$

where $q_{g e n}^{\prime \prime}$ is the heat flux generated in the foil by Joule heating given by Eq 3. The second term on the RHS accounts for heat lost across the air gap cavity and is given by Eq 4, where $k_{a g}$ is the thermal conductivity of the bulk fluid (air) and $\mathrm{dT} / \mathrm{dz}$ is the temperate gradient across the air gap to the IR window. $q_{r a d, b}^{\prime \prime}$ is the total radiative emission from the underside of the foil to the top of the IR transparent $\mathrm{CaF}_{2}$ glass and is given by $\mathrm{Eq}$ 5. The fourth term in Eq 2 refers to heat transfer due to lateral conduction given by Eq 6 . The final term accounts for the energy stored within the foil.

$$
\begin{gathered}
q_{g e n}^{\prime \prime}=\frac{I_{s} V_{s}}{A_{s}} \\
q_{c o n d}^{\prime \prime}=-k_{a g} \frac{d T}{d z} \\
q_{r a d, b}^{\prime \prime}=\frac{\sigma\left(T_{s}^{4}-T_{C a F_{2}}^{4}\right)}{1 / \varepsilon_{p}+1 / \varepsilon_{C a F_{2}}-1} \\
q_{l c}^{\prime \prime}=-\left(k_{f} \delta_{f}+k_{p} \delta_{p}\right)\left(\frac{\partial^{2} T_{s}}{\partial x^{2}}+\frac{\partial^{2} T_{s}}{\partial y^{2}}\right) \\
q_{c a p}^{\prime \prime}=\left(\rho_{f} C_{p, f} \delta_{f}+\rho_{p} C_{p, p} \delta_{p}\right) \frac{\partial T_{s}}{\partial t}
\end{gathered}
$$




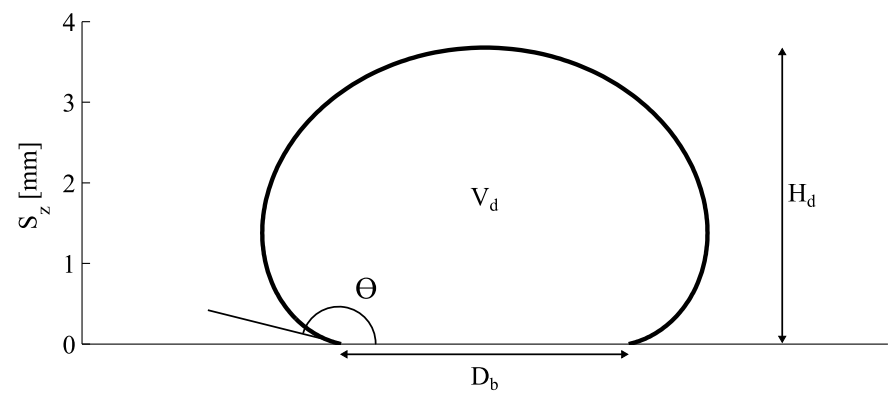

Figure 5: Schematic of droplet geometric parameters.

\section{Averaging}

The final stage of processing is to average all images over each test. This assumes that the droplet evaporation is quasi-steady during the 1 second capture period. As will be discussed, this assumption is shown to be valid due to the relatively low calculated energy storage term. The radial profile of the convective heat flux is then calculated by taking a line radially from the centre of the convective heat flux map in $0.5^{\circ}$ increments and averaging the results.

\subsubsection{Optical images processing}

The captured optical data were processed using custom built MATLAB code. Image processing was broken down into two phases: boundary detection and filtering and image analysis.

\section{Boundary detection and filtering}

The captured image was converted to grayscale and a no droplet background image was subtracted. Then the image was binarised, sensitised, and filled. The detected boundary was then broken up into evenly spaced segments and filtered using a Savitzky - Golay filter.

\section{Image analysis}

From the filtered boundary a number of key parameters such as; droplet height $H_{d}$, base radius, volume $V_{d}$, centroid, contact angle, interfacial areas and radii of curvature can be calculated. These parameters are shown in Fig. 5. The local curvature of the droplet at any position, $C$, is given by:

$$
2 C=\frac{1}{r_{1}}+\frac{1}{r_{2}}
$$

The local mechanical equilibrium between each point of the droplet liquid-gas interface can be expressed by the Young-Laplace equation. Accounting for only hydrostatic effects this equation is given by:

$$
2 C=\frac{2 \gamma}{r_{T}}-g\left(\rho_{l}-\rho_{v}\right)\left(z-z_{0}\right)
$$

where $z_{0}$ is the reference height, taken as the droplet height and $z$ is the height of the point on the interface being evaluated. This equation evaluates the balance between the pressure difference across the interface and the stress in the

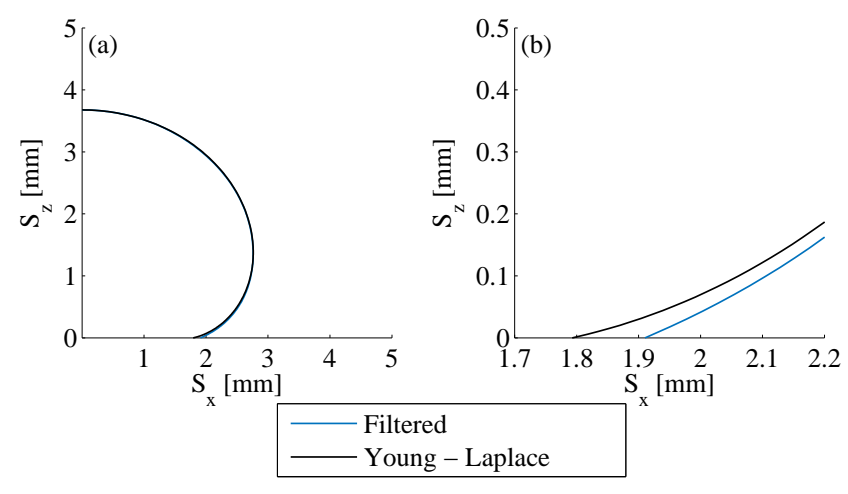

Figure 6: Filtered boundary compared with Young-Laplace solution.

interface, expressed as the product of the surface tension and the curvature. Due to droplet reflections, shadowing and the mirage effect arising from the evaporating droplet at the base, the droplet shape near the triple line can be distorted. For the hydrostatic only case it is possible, from experimental data, to calculate the droplet shape according to the Young-Laplace equation. Similar to Siedel et al. [39] an iterative method was employed to calculate the droplet interface profile. The method requires the input of the apex curvature, droplet height and droplet volume. The equation was solved for the initial apex curvature and the volume of the produced Young-Laplace curve was compared with that of the experimental data. The tip curvature was then adjusted based on this volume ratio. This process continues until the Young-Laplace solution volume converges to that of the experimental data. A difference of $\pm 0.01 \mu \mathrm{L}$ was set as the threshold difference. As the experimental boundary may be distorted at the base only the upper $95 \%$ of the Young-Laplace and experimental boundaries are compared. A comparision of experimental data and the boundary calculated from the Young-Laplace equation is shown in Fig. 6.

\subsection{Uncertainty Analysis}

The experimental uncertainty for all parameters was examined using the methodology outlined by Kirkup and Frenkel [40]. A list of the relevant parameter and their associated percentage uncertainty (PU) is outlined in Ta-

Table 3: Droplet evaporation experimental uncertainty.

\begin{tabular}{lcr}
\hline Parameter & & $\mathrm{PU}[\%]$ \\
\hline$T_{s}$ & \pm & 0.25 \\
$T_{\infty}$ & \pm & 0.04 \\
$T_{a g}$ & \pm & 0.04 \\
$q_{g e n}^{\prime \prime}$ & \pm & 1.97 \\
$V_{d}$ & \pm & 5.31 \\
$\theta$ & \pm & 34.09 \\
$D_{b}$ & \pm & 2.69 \\
$H_{d}$ & \pm & 1.52 \\
$r_{T}$ & \pm & 2.54 \\
\hline
\end{tabular}


ble 3. All listed values are to a $95 \%$ confidence level. First the standard uncertainty ( $\mathrm{SU}$ ) of the acquired test data is determined. A combined uncertainty approach is then applied on a pixel by pixel basis to Eq 2-7. The expanded uncertainty of a single sample of an individual pixel recorded by the IR camera is $\pm 0.77 \mathrm{~K}$ for this experimental study. This value is determined from the calibration curve fit, accounting for the uncertainty attached to the polynomial fit between counts (IR camera data aquisition) and temperature. The expanded uncertainty for the radial profile of $q_{d e}^{\prime \prime}, q_{l c}^{\prime \prime}, q_{c a p}^{\prime \prime}, q_{r a d, b}^{\prime \prime}$, and $q_{c o n d}^{\prime \prime}$ are shown graphically in Fig. 7. These values are highlighted at three key points on the radial profile.

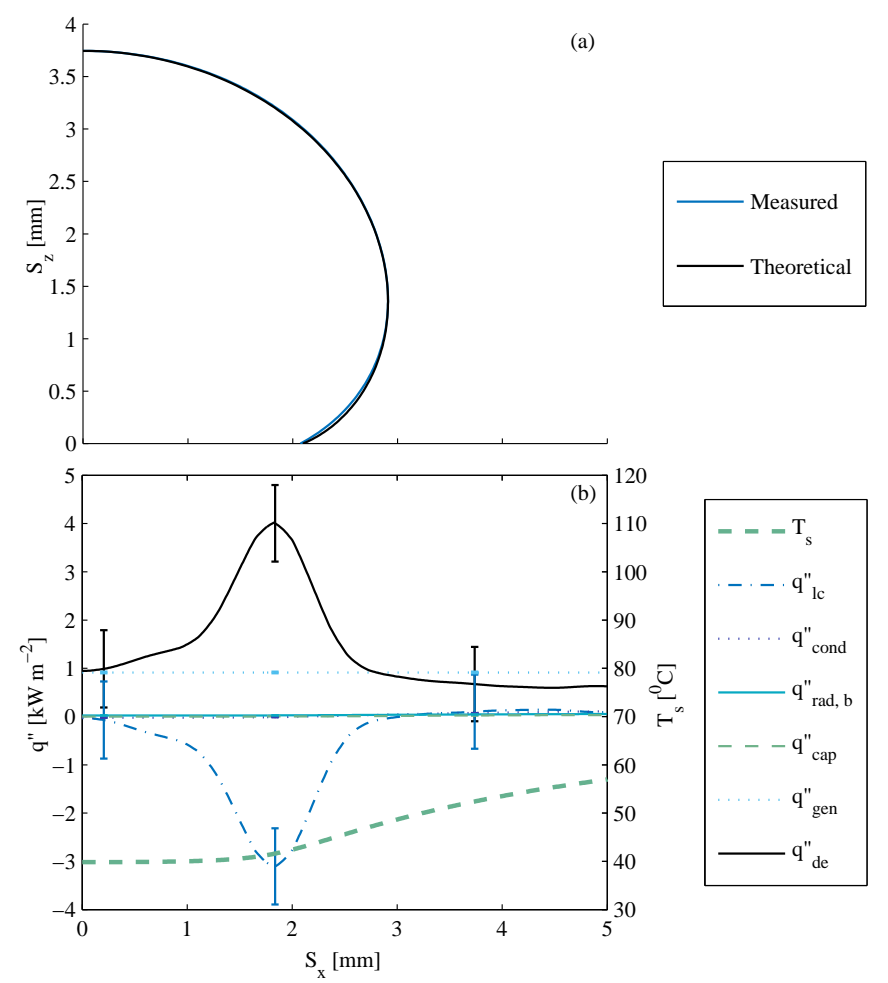

Figure 7: Partial non-wetting droplet. (a) liquid-gas interface and (b) local heat flux energy balance at $\mathrm{t}=2 \mathrm{~min}$.

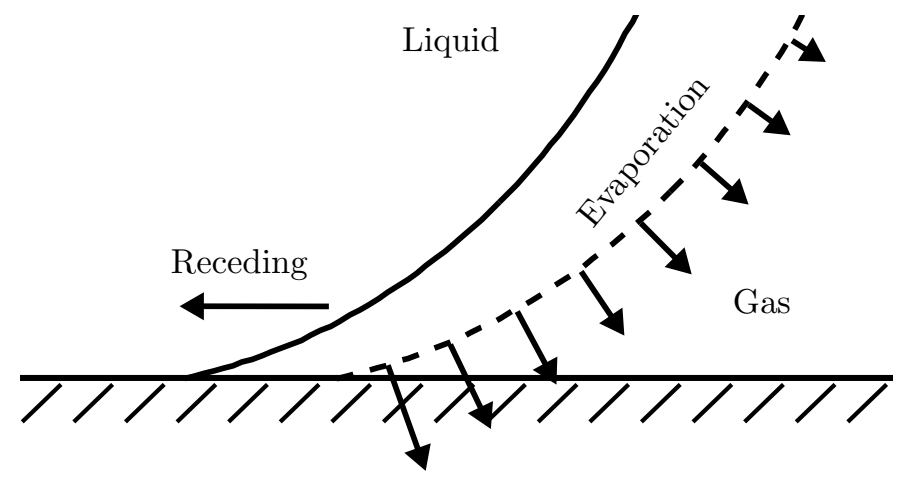

Figure 8: Superhydrophobic CCA droplet evaporation at the contact line.

\section{Results and Discussion}

Fig. 7 compares the liquid-gas interface captured by the optical camera (Fig. 7a) with the radial heat flux energy balance calculated from data acquired by the IR camera at $t=2 \mathrm{~min}$. A peak local heat flux value of $4.02 \mathrm{~kW} \mathrm{~m}^{-2}$ is noted at $S_{x}=1.83 \mathrm{~mm}$. This peak heat flux is located at the droplet triple line. This resembles the surface heat flux profile previously observed by Karchevsky et al. [32] and Gibbons et al. [41] for their hydrophilic droplets. It is worth noting that this result also resembles those provided by Gibbons and Robinson [13] on the same test facility, albeit for highly wetting ethanol droplets formed by electrospray. Examining the energy balance terms it is noted that the largest contribution is due to lateral conduction. Lateral conduction accounts for energy transfer within the foil. Total droplet evaporation was completed by $t=32$ min. Fig. 9 shows the 3D droplet evaporation process at three different stages. The droplet evaporated in a CCA regime over the presented time scale. The highest heat flux is observed at the triple contact line for all time points.

As discussed earlier, for hydrophilic droplets the peak heat transfer occurs at the contact line region due to the low thermal resistance of the thin liquid film that forms between the bulk droplet and the adsorbed film regions. For the present superhydrophobic droplet evaporating in CCA mode, it is clear that a peak surface heat flux once
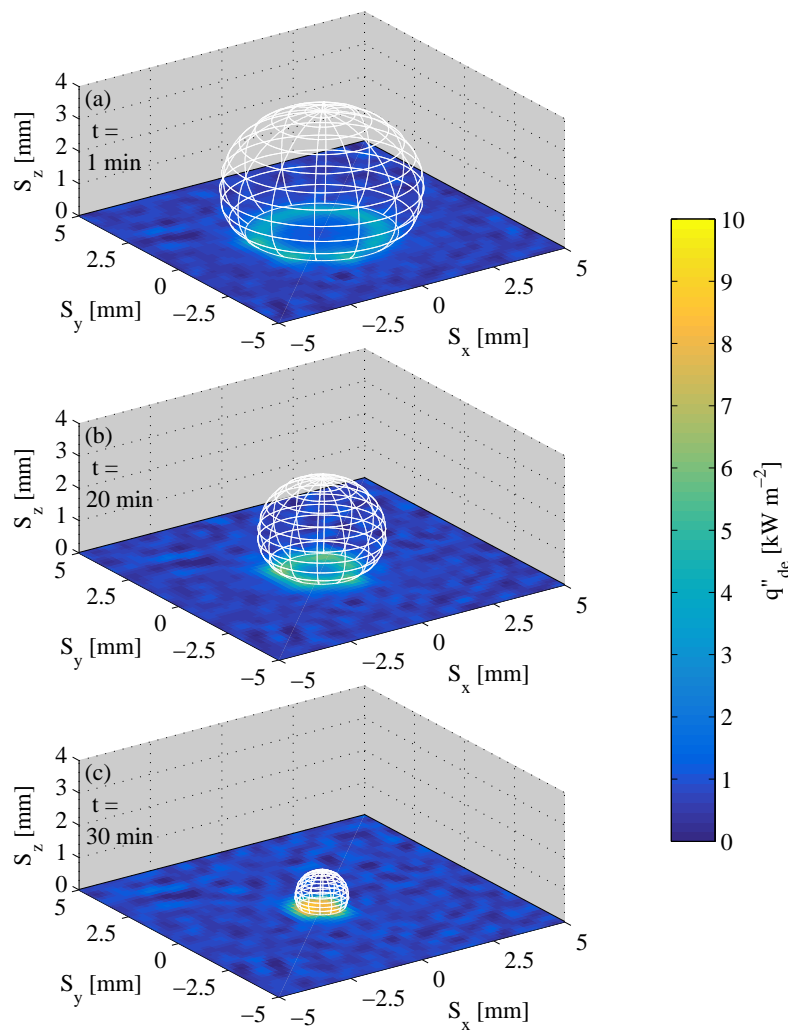

Figure 9: Partial non-wetting droplet evaporation. (a) $\mathrm{t}=1 \mathrm{~min}$, (b) $\mathrm{t}=20 \mathrm{~min}$, and (c) $\mathrm{t}=30 \mathrm{~min}$. Total evaporation time $\approx 32$ min. 
again occurs at the contact line region, and to the best of knowledge this is the first time that this has been observed for non-wetting droplets. This indicates that a low thermal resistance pathway between the heated surface and the liquid-gas interface, where diffusion driven evaporation occurs, must exist in this region. It is proposed here that the mechanism is a straight forward extension to that described by Pan et al. [34]. The main difference between this study and the numerical investigation of Pan et al. [34] is that the latter considered free evaporation and the evaporative cooling effect at the interface induced thermal gra- dients within the system, something which is not considered in isothermal free evaporation models. They found that the now relatively hot surface, compared with the cooled tip of the droplet, caused there to be a hotter region of the droplet interface near the contact line and this corresponded to a higher saturation pressure and vapour fraction gradient which leads to a high evaporation flux. Thus, for the scenario considered here where the surface is heated, one would expect that this mechanism would be enhanced due to the higher local interface temperatures associated with the heated surface. Simply put, a thermal
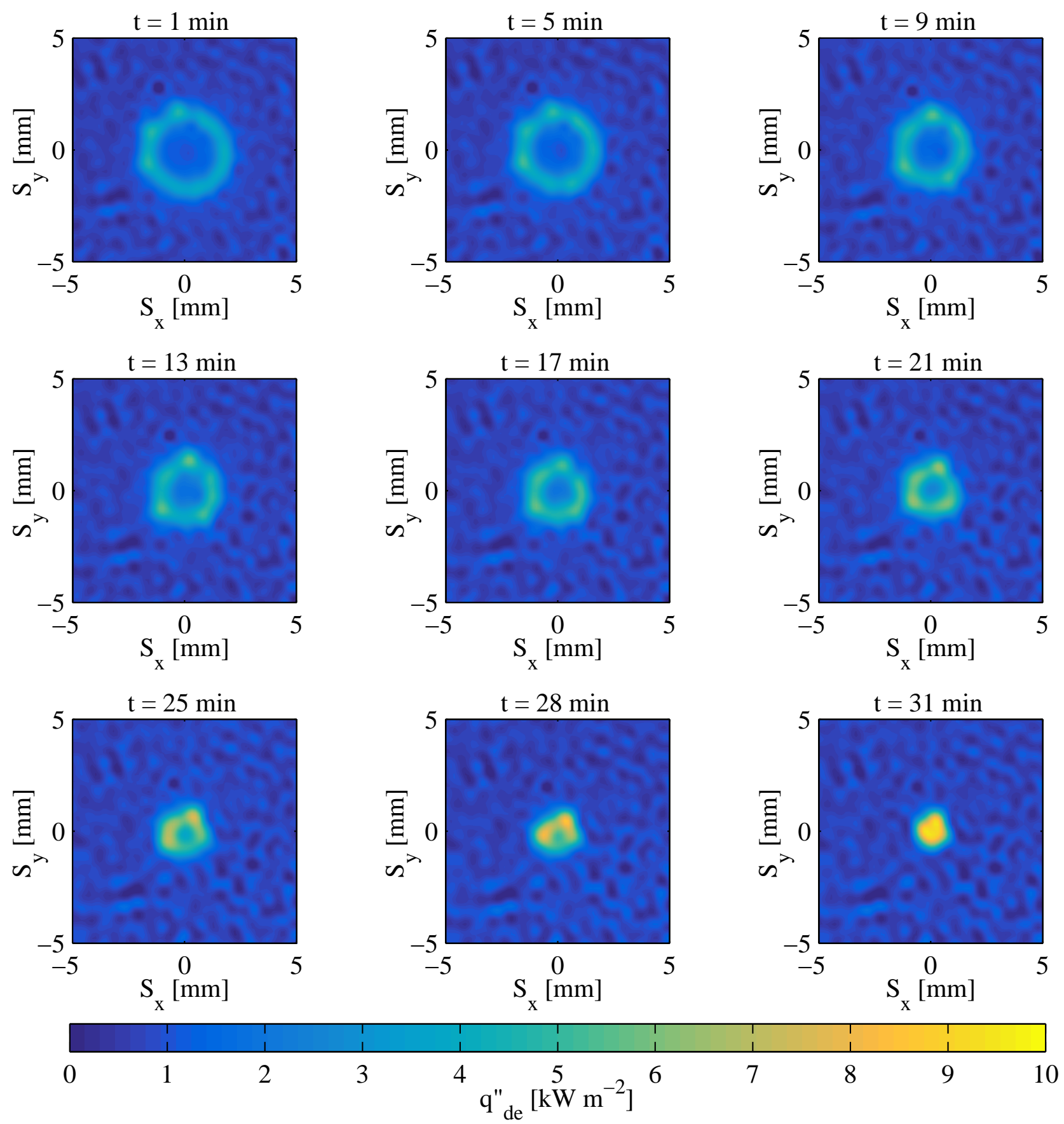

Figure 10: Partial non-wetting droplet evaporation heat flux distribution over time. 
boundary layer is established in the liquid in the region of the heated surface, and the local thickness of the thermal boundary layer dictates the local thermal resistance and thus substrate surface heat flux. Near the contact line, the thermal boundary layer intersects the liquid-gas interface creating a region below which the liquid-gas interface temperature is high. Here, diffusion and possibly advection of heat to the droplet interface is released effectively due to the proportionately higher saturation pressure. Therefore, the close proximity of the heated surface and the liquidgas interface in the contact line region combined with the high vapour diffusion to the ambient, creates an overall low thermal resistance pathway between the heat source (heated substrate) and the heat sink (the ambient air) and this causes the observed peak surface heat flux in this region. Of course, conservation of mass requires that liquid from the bulk droplet must be advected to the contact line region to feed that which is being evaporated, so it is quite likely that the thermal boundary layer will be thinned to some degree which will would also influence the surface heat transfer. Also, compared with the simulation study of Pan et al. [34] where the droplet is static and geomet- ric confinement is noted to impede diffusion of vapour to the ambient, the droplet studied here evaporates in CCA mode with a receding contact line, as depicted in Fig. 8. $\mathrm{Xu}$ et al. [30] noted that for free evaporation of droplets on superhydrophobic surfaces in CCA mode, the inward motion of the contact line helps mitigate the accumulation of vapour in the contact line region i.e. contact line motion helps reduce the vapour concentration in the wedge-shaped contact line region. The relative importance of these last two mechanisms discussed requires detailed simulations to be carried out and is outside the scope of the present study.

Fig. 10 highlights the local heat flux distribution of the partial non-wetting evaporating droplet over time. The droplet is observed to evaporate with a receding contact line. This is due to the low contact angle hysteresis. The solid-liquid interface is shown to decrease, while the magnitude of the heat flux increases. The droplet is considered axisymmetric while the the deviation of the multiple radial lines taken at $0.5^{\circ}$ intervals from the droplet centre to droplet edge is within $10 \%$ of the average droplet base radius. This condition is true for $t=1-25 \mathrm{~min}$. At the end of the evaporation ( $t=28-32 \mathrm{~min}$ ) the contact line
$1 \mathrm{~min}$

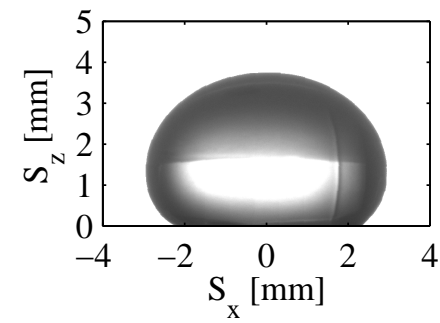

$15 \min$

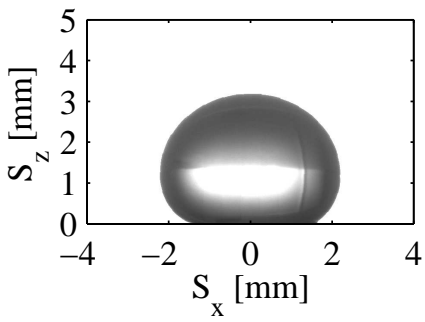

$25 \min$

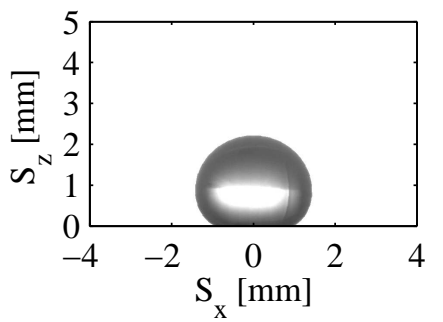

$31 \min$

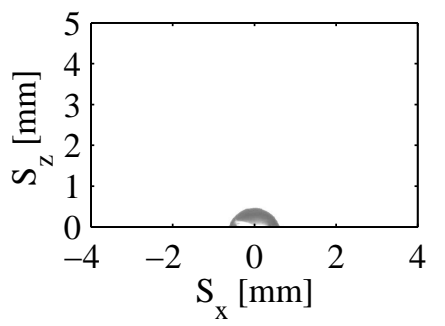

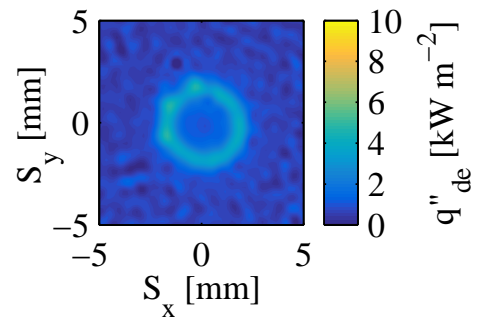
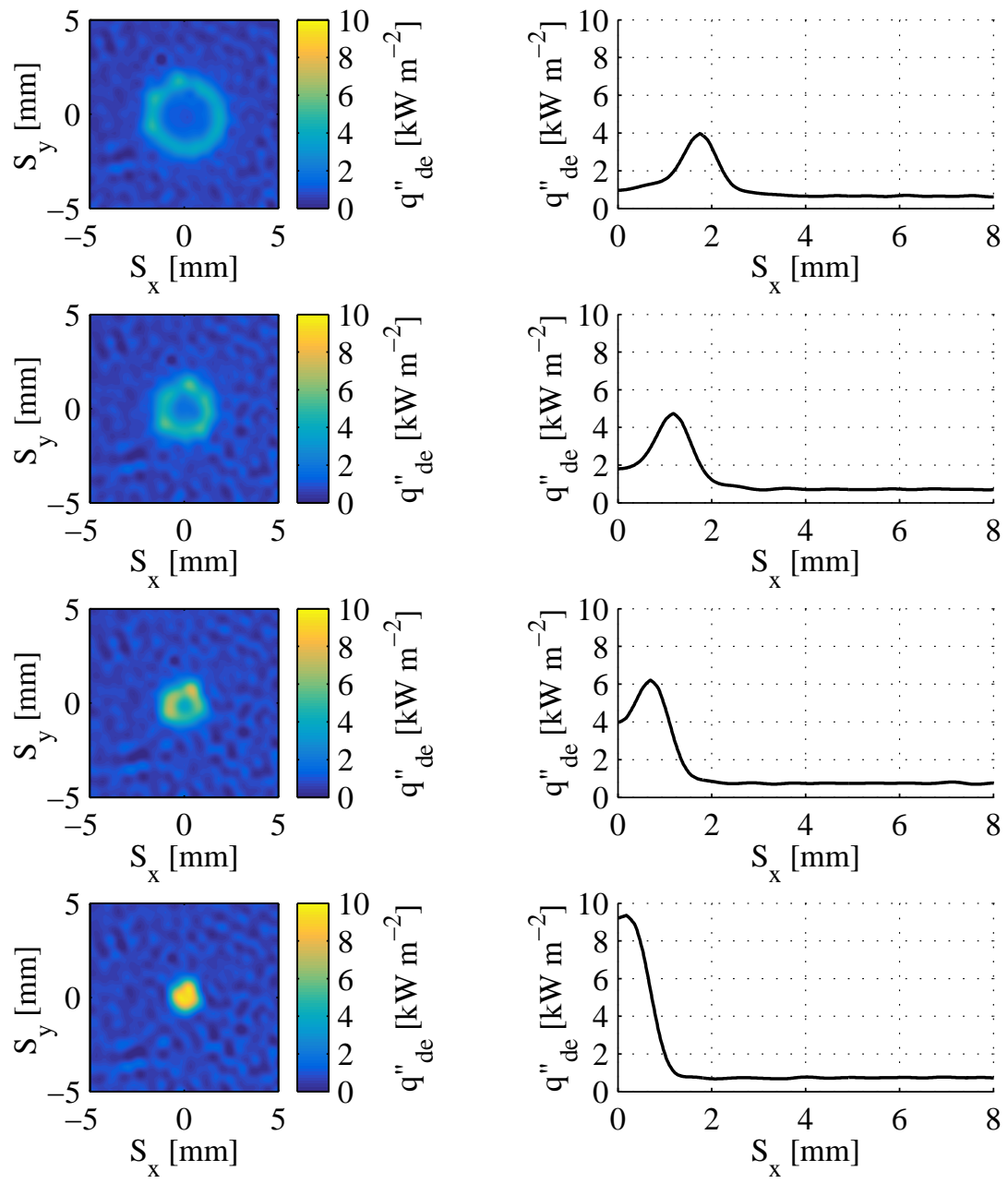

Figure 11: Partial non-wetting droplet evaporation, shape, heat flux distribution, and radial heat flux profile over time. 
unpins in a stochastic manner to final complete droplet evaporation at $t=32 \mathrm{~min}$. Droplet evaporation for $t<$ $1 \mathrm{~min}$ is not considered here as the system behaves in a transient manner before this point as a result of the step input of heat into the foil and subsequent transients in temperature beginning at $t=0 \mathrm{~min}$.

Fig. 11 shows the droplet morphology, convective heat flux distribution and local convective radial heat flux profiles of the evaporating droplet at four different stages of the evaporation process. The droplet is qualitatively ob- served to evaporate with a decreasing droplet base and height. The plots of the radial heat flux profile show that the location of the peak heat flux tracks with the receding triple line and increases in magnitude as the droplet evaporates. As will be discussed this is an interesting result in the context of thermal power dissipation to the droplet since the decreasing liquid - solid interface area is partially offset by the increase convective heat flux. This is due to
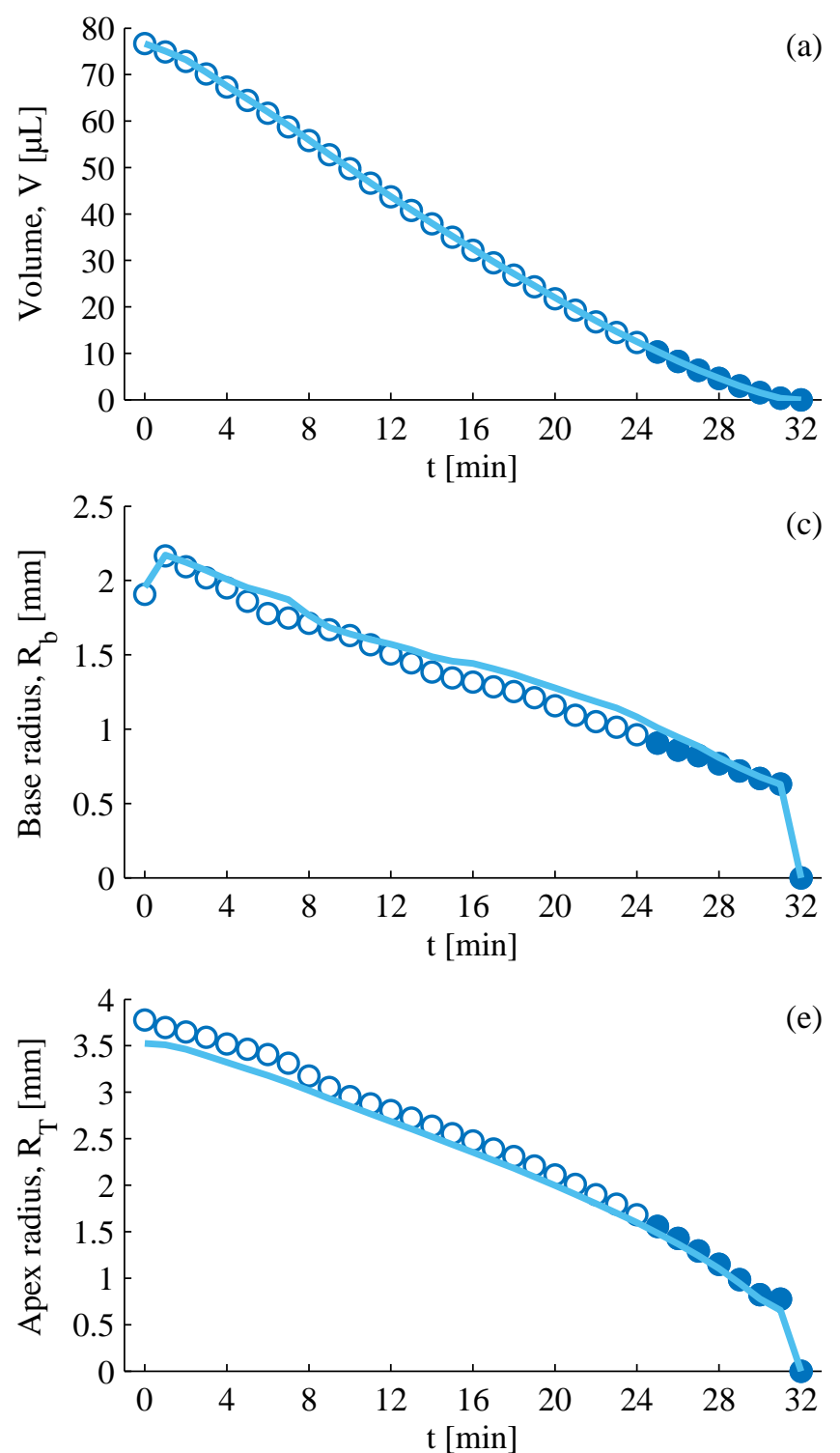
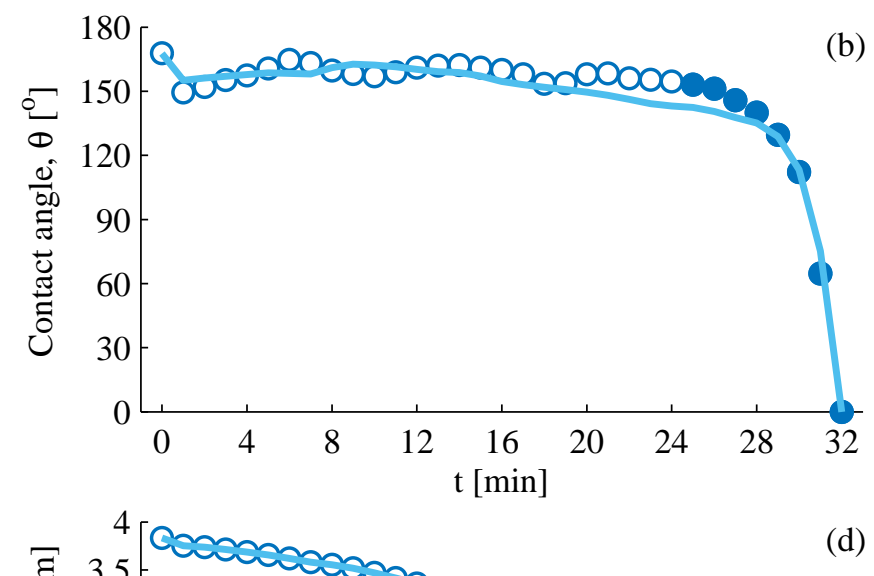

(d)

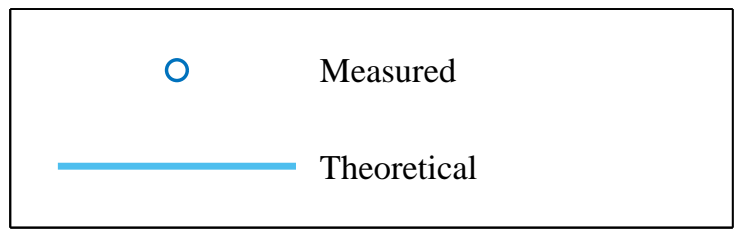

Figure 12: Partial non-wetting droplet geometric properties through time. (a) volume, (b) contact angle, (c) base radius, (d) height, (e) apex principal radii, and (f) liquid-gas surface area. Empty markers = axisymmetric droplet, filled markers $=$ non-axisymmetric droplet. 
the increasing contact line length density ${ }^{2}$ (CLL) and surface temperature of the solid-liquid interface.

Fig. 12 details how various geometric properties of the droplet vary over the entire evaporation period. Results from both the measured and the Young-Laplace solution are shown for comparison. The largest discrepancy between the measured interface and the Young-Laplace solution is in the contact angle. This stems from the aforementioned difficulties measuring the triple line region of the evaporating droplet. The filled markers denote when the droplet is no longer considered axisymmetric. The droplet is observed to initially evaporate in the CCA regime. From $t=1-28$ min the droplet evaporated with a relatively constant contact angle of $\approx 150^{\circ}$. The CCA mode of evaporation is considered while the deviation of instantaneous contact angle is within 10\% of the receding contact angle of the droplet. This is similar to the approach and results of Dash and Garimella [42] for their natural evaporation of hydrophobic and superhydrophobic droplets. All parameters, except the contact angle, are generally observed to decrease over the course of the droplet evaporation. The near constant contact angle, decreasing droplet radius and height give credence to the CCA evaporation regime. Near the end of the evaporation process $(t=29-32 \mathrm{~min})$, the contact angle and contact radius are observed to decrease simultaneously to complete evaporation. This mixed mode

${ }^{2} \mathrm{CLL}=$ contact line length / wetted area
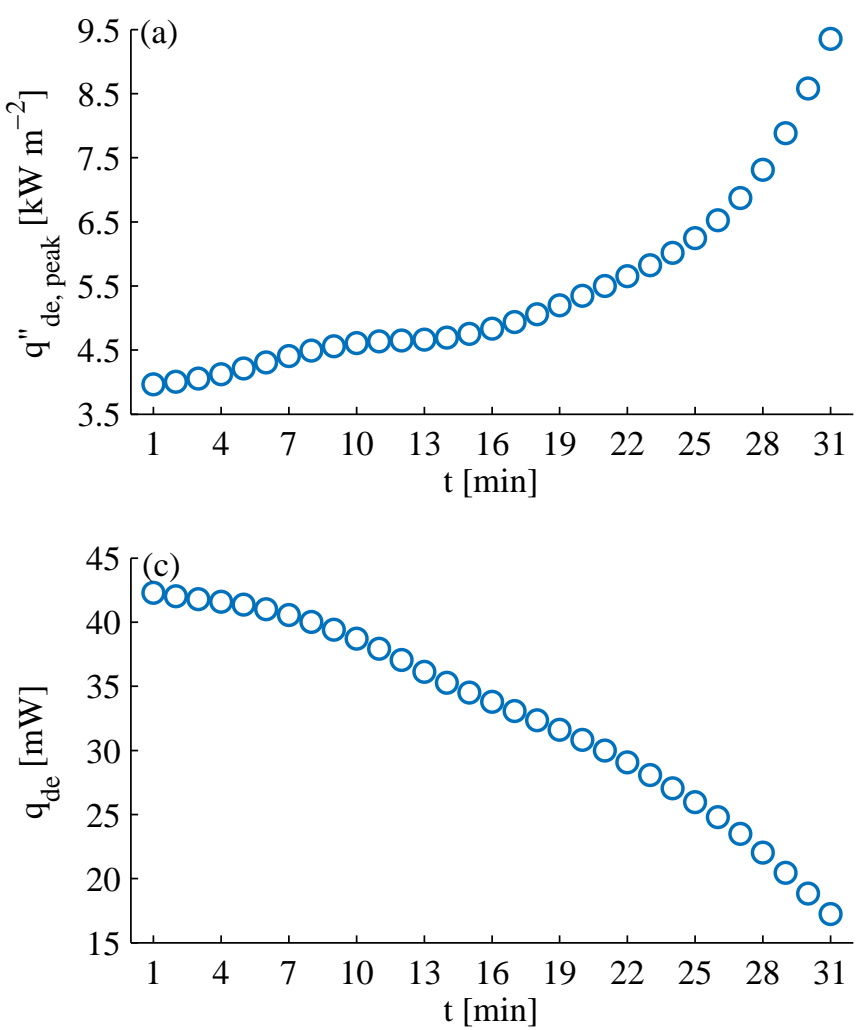

evaporation consists of $10 \%$ of the total evaporation time. Thus, the droplet evaporation is predominantly CCA. The trends and shape for contact angle, droplet height and base radius are in good agreement with those observed by Dash and Garimella [42].

Figs 13a show the magnitude of the peak local heat flux to the evaporating droplet, which is located at the triple line. An escalating increase in $q_{d e, p e a k}^{\prime \prime}$ is noted over the evaporation period. This is due to the increasing CLL as the droplet evaporates. Figs 13b demonstrates the change in the solid-liquid interfacial area. A generally monotonically decreasing area is observed. Figs $13 \mathrm{c}$ and d plot the total thermal power and the average heat flux into the droplet across the solid-liquid interface. Decreasing values of $q_{d e}$ and increasing values of $q_{d e, a v e}^{\prime \prime}$ are noted. The trend in $q_{d e}$ correlates closely with that observed by Sobac and Brutin [15] for their unpinned natural evaporation of ethanol and toluene on a PTFE coated isothermal substrate. The result of increasing $q_{d e, a v e}^{\prime \prime}$ with increasing CLL is in good agreement with that observed by Crafton and Black [43] and Gatapova et al. [44] for their unpinned heated substrate evaporation of n-heptane and water droplets respectively. Good correlation of the trend of $q_{d e, p e a k}^{\prime \prime}$ is observed for receding contact line to that shown by Gibbons and Robinson [13] during their single source electrospray cooling study. In the pool cooling regime as the flow rate is decreased the quasi-steady droplet on the heated surface reduces in size and subsequently the
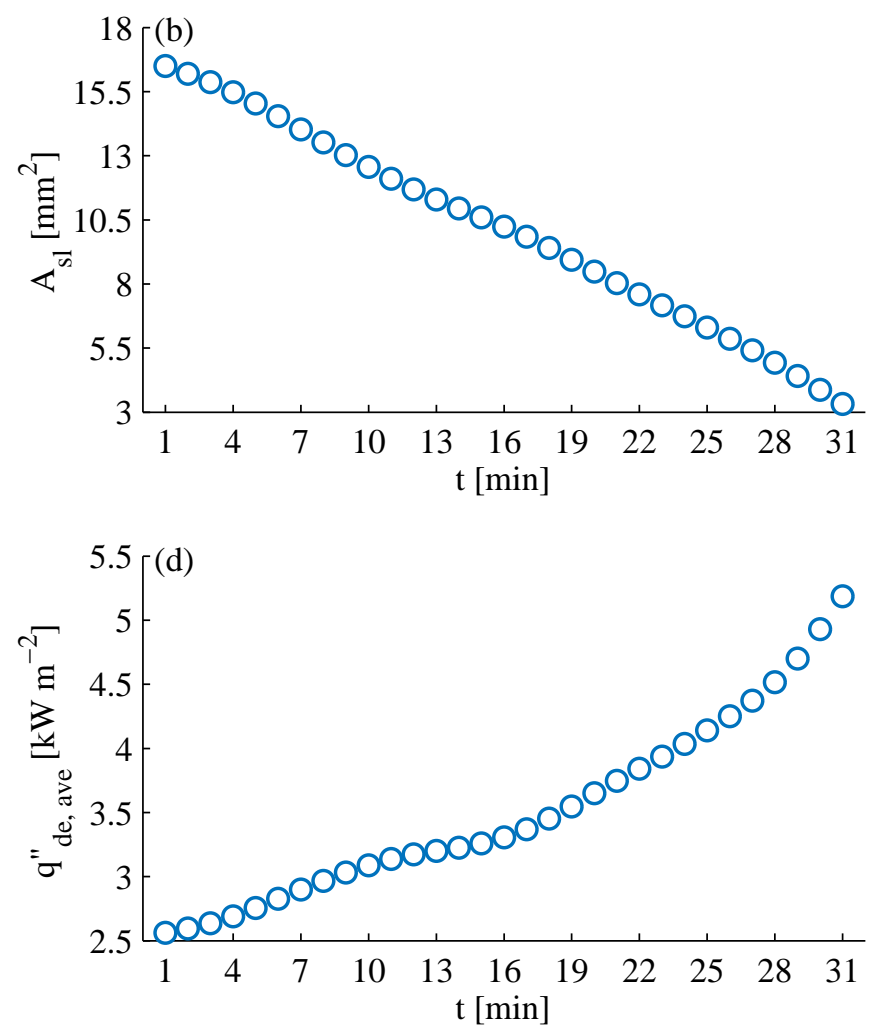

Figure 13: Partial non-wetting droplet evaporation heat transfer. (a) peak heat flux magnitude, (b) solid-liquid surface area, (c) total power into droplet, and (d) average heat flux. 


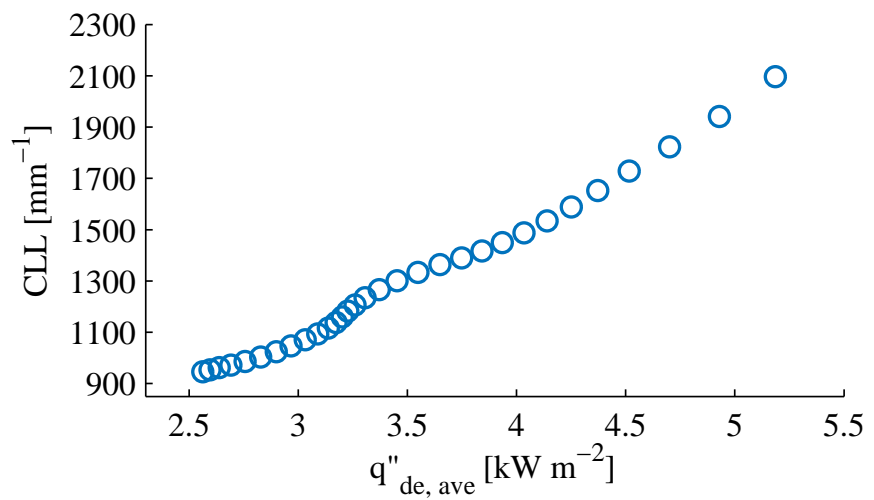

Figure 14: Average heat flux to the evaporating droplet compared with the contact line density over the complete evaporation period.

wetted area decreases and CLL increases. This resulted in a similar increase in the peak heat flux at the contact line.

As the droplet evaporates in the CCA regime the surface area between the solid substrate and liquid droplet decreases. As this occurs the height of the droplet also decreases. This reduction in the solid-liquid and liquid-gas interface results in a reduction in the total thermal power into the droplet. However an increase in the heat flux into the droplet is observed due to the increasing temperature of the liquid-gas interface and CLL. Thus, the rate at which the heat transfer due to the decreasing area of heat transfer is partially offset by the increasing convective heat flux. The relationship between the CLL and the average heat heat flux into the droplet is further explored in Fig. 14.

Fig. 14 demonstrates the importance on the CLL in relation to the average heat flux removed to an evaporating droplet. A linear relationship between the CLL and the average heat flux is observed. This result highlights the greater importance of the CLL over the liquid-gas surface area. This linear trend is in agreement with that observed by Horacek and Kim [45] and Sodtke and Stephan [46] during their spray cooling studies. This outcome also supports the conclusions of Gibbons and Robinson [13]. They noted that peak heat transfer was achieved for maximum CLL when the generated droplets impinge on the heated substrate, forming multiple individual contact lines, and evaporate.

Fig. 15 presents the droplet liquid interface (a) and the radial heat flux profile (b) over time onto individual plots. This figure succinctly shows the droplet evaporation dynamics discussed previously. The CCA evaporation regime is shown with its moving triple line evaporation. The decreasing area of convective heat transfer is accompanied by an increase in the convective heat flux. It is noteworthy that as the droplet thickness decreases the local heat flux around $S_{x}=0 \mathrm{~mm}$ increases. This can be attributed to the increasing liquid-gas interface temperature, due to the decreasing thermal resistance, as the droplet evaporates. In the droplet macro region, the smaller liquid-gas surface area at a higher temperature $(\mathrm{t}=31 \mathrm{~min})$ results
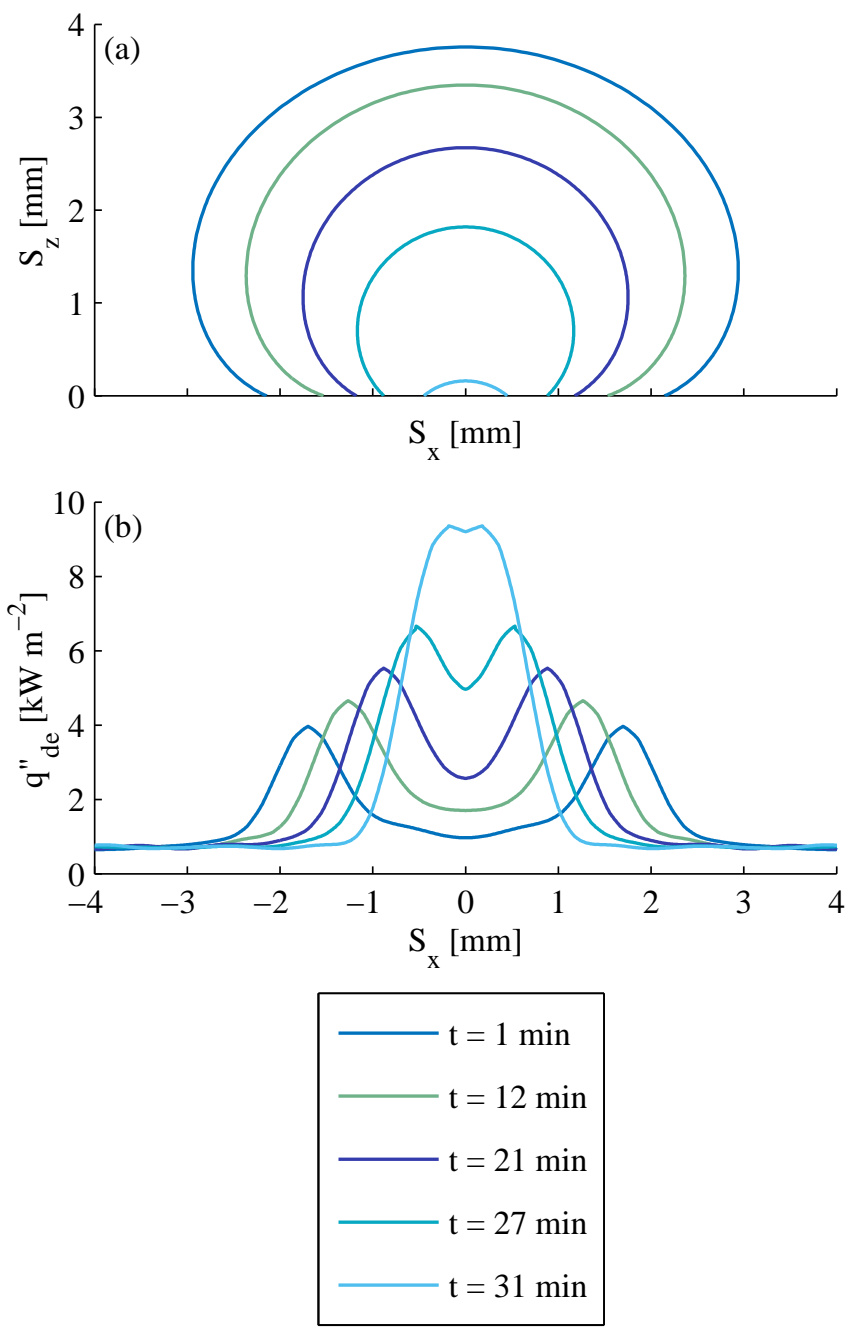

Figure 15: Partial non-wetting droplet evaporation. (a) liquid-gas interface and (b) convective heat flux profile.

in a great thermal dissipation than the larger solid-liquid surface area $(\mathrm{t}=1 \mathrm{~min})$ at a lower temperature.

\section{Conclusion}

The convective heat flux distribution beneath a partially non-wetting evaporating droplet with low contact angle hysteresis was studied using thin foil thermography and optical image processing. The droplet evaporated in the CCA regime. The peak heat transfer was noted at the triple contact line for the all test points. It is believed that this is the first time that the enhanced heat transfer at the contact line region for superhydrophobic evaporating droplets has been observed experimentally. This result confirms the assertion by Pan et al. [34] that conjugate heat transfer must be considered when analysing evaporating droplets as it establishes a elevated temperature region of high evaporative mass flux from the droplet in the vicinity of the contact line, and this is true for both free evaporation and evaporation on a heated substrate. 
The peak and average heat flux were shown to increase as the evaporation proceeded. This was due to the increasing CLL, as a result of the receding contact line, and the increasing liquid-gas interface temperature, due to the decreasing droplet thickness. The total thermal power across the solid-liquid interface decreased as the evaporation proceeded, due to the decreasing solid-liquid surface area. The average heat flux across the solid-liquid boundary was shown to vary linearly with the CLL. This research demonstrates the importance of the contact line in droplet evaporation. It has direct application in numerous field, most pertinently that of spray cooling where optimum heat transfer is achieved by maximising the CLL. Future work will involve evaporation of a partially wetting droplet with large contact angle hysteresis as well as the influence of electric fields and microgravity on droplet evaporation dynamics and heat transfer.

\section{Acknowledgements}

The authors would like to acknowledge the financial support of the Irish Research Council under Grant No. RS/2012/86. We would also like to thank Dr Jason Reese (University of Edinborough), Dr Jun Zhang (Beihang University), Dr Feng-Chao Wang (University of Science and Technology of China) and Dr David Brutin (Ecole Polytechnique Universitaire de Marseille) and Dr Alex Rednikov (Universit'e Libre de Bruxelles) for their useful insights and discussions.

\section{References}

[1] S. A. Putnam, A. M. Briones, J. S. Ervin, M. S. Hanchak, L. W. Byrd, J. G. Jones, Interfacial heat transfer during microdroplet evaporation on a laser heated surface, International Journal of Heat and Mass Transfer 55 (23) (2012) 6307-6320.

[2] W. Deng, A. Gomez, Electrospray cooling for microelectronics, International Journal of Heat and Mass Transfer 54 (11) (2011) $2270-2275$.

[3] T. J. Hartranft, G. S. Settles, Sheet atomization of nonnewtonian liquids, Atomization and Sprays 13 (2\&3).

[4] M. Schena, D. Shalon, R. W. Davis, P. O. Brown, Quantitative monitoring of gene expression patterns with a complementary DNA microarray, Science 270 (5235) (1995) 467.

[5] V. Dugas, J. Broutin, E. Souteyrand, Droplet evaporation study applied to DNA chip manufacturing, Langmuir 21 (20) (2005) 9130-9136.

[6] A. Boutier, Laser Metrology in Fluid Mechanics: Granulometry, Temperature and Concentration Measurements, John Wiley \& Sons, 2013.

[7] G. Li, S. M. Flores, C. Vavilala, M. Schmittel, K. Graf, Evaporation dynamics of microdroplets on self-assembled monolayers of dialkyl disulfides, Langmuir 25 (23) (2009) 13438-13447.

[8] J. Kim, Spray cooling heat transfer: the state of the art, International Journal of Heat and Fluid Flow 28 (4) (2007) 753-767.

[9] S. A. Putnam, A. M. Briones, L. W. Byrd, J. S. Ervin, M. S. Hanchak, A. White, J. G. Jones, Microdroplet evaporation on superheated surfaces, International Journal of Heat and Mass Transfer 55 (21) (2012) 5793-5807.

[10] R.-H. Chen, T. X. Phuoc, D. Martello, Effects of nanoparticles on nanofluid droplet evaporation, International Journal of Heat and Mass Transfer 53 (19) (2010) 3677-3682.
[11] J. Park, J. Moon, Control of colloidal particle deposit patterns within picoliter droplets ejected by ink-jet printing, Langmuir 22 (8) (2006) 3506-3513.

[12] D. Brutin, Droplet Wetting and Evaporation: From Pure to Complex Fluids, Academic Press, 2015.

[13] M. Gibbons, A. Robinson, Heat transfer characteristics of single cone-jet electrosprays, International Journal of Heat and Mass Transfer 113 (2017) 70-83.

[14] R. G. Picknett, R. Bexon, The evaporation of sessile or pendant drops in still air, Journal of Colloid and Interface Science 61 (2) (1977) 336-350.

[15] B. Sobac, D. Brutin, Triple-line behavior and wettability controlled by nanocoated substrates: influence on sessile drop evaporation, Langmuir 27 (24) (2011) 14999-15007.

[16] S. Semenov, V. Starov, R. Rubio, H. Agogo, M. Velarde, Evaporation of sessile water droplets: Universal behaviour in presence of contact angle hysteresis, Colloids and Surfaces A: Physicochemical and Engineering Aspects 391 (1) (2011) 135-144.

[17] S. David, K. Sefiane, M. E. Shanahan, On the dynamics of wetting of water-methanol volatile sessile drops on smooth substrates, in: Defect and Diffusion Forum, Vol. 258, Trans Tech Publ, 2006, pp. 469-473.

[18] S. David, K. Sefiane, L. Tadrist, Experimental investigation of the effect of thermal properties of the substrate in the wetting and evaporation of sessile drops, Colloids and Surfaces A: Physicochemical and Engineering Aspects 298 (1) (2007) 108114.

[19] G. Dunn, S. Wilson, B. Duffy, S. David, K. Sefiane, A mathematical model for the evaporation of a thin sessile liquid droplet: Comparison between experiment and theory, Colloids and Surfaces A: Physicochemical and Engineering Aspects 323 (1) (2008) 50-55.

[20] V. Vancauwenberghe, P. Di Marco, D. Brutin, Wetting and evaporation of a sessile drop under an external electrical field: A review, Colloids and Surfaces A: Physicochemical and Engineering Aspects 432 (2013) 50-56.

[21] P. A. Raghupathi, S. G. Kandlikar, Contact line region heat transfer mechanisms for an evaporating interface, International Journal of Heat and Mass Transfer 95 (2016) 296-306.

[22] I. Marchuk, A. Karchevsky, A. Surtaev, O. Kabov, Heat flux at the surface of metal foil heater under evaporating sessile droplets, International Journal of Aerospace Engineering 2015.

[23] A. Cazabat, G. Guena, Evaporation of macroscopic sessile droplets, Soft Matter 6 (12) (2010) 2591-2612.

[24] K. Gleason, H. Voota, S. A. Putnam, Steady-state droplet evaporation: Contact angle influence on the evaporation efficiency, International Journal of Heat and Mass Transfer 101 (2016) $418-426$.

[25] F. Carle, B. Sobac, D. Brutin, Experimental evidence of the atmospheric convective transport contribution to sessile droplet evaporation, Applied Physics Letters 102 (6) (2013) 061603.

[26] J. L. Plawsky, A. G. Fedorov, S. V. Garimella, H. B. Ma, S. C. Maroo, L. Chen, Y. Nam, Nano- and microstructures for thinfilm evaporation-A review, Nanoscale and Microscale Thermophysical Engineering 18 (3) (2014) 251-269.

[27] S. Dash, S. V. Garimella, Droplet evaporation on heated hydrophobic and superhydrophobic surfaces, Physical Review E 89 (4) (2014) 042402.

[28] R. D. Deegan, O. Bakajin, T. F. Dupont, G. Huber, S. R. Nagel, T. A. Witten, Capillary flow as the cause of ring stains from dried liquid drops, Nature 389 (6653) (1997) 827-829.

[29] Z. Zheng, L. Zhou, X. Du, Y. Yang, Numerical investigation on conjugate heat transfer of evaporating thin film in a sessile droplet, International Journal of Heat and Mass Transfer 101 (2016) 10-19.

[30] W. Xu, R. Leeladhar, Y. T. Kang, C.-H. Choi, Evaporation kinetics of sessile water droplets on micropillared superhydrophobic surfaces, Langmuir 29 (20) (2013) 6032-6041.

[31] M. Shanahan, Simple theory of stick-slip wetting hysteresis, Langmuir 11 (3) (1995) 1041-1043.

[32] A. L. Karchevsky, I. V. Marchuk, O. A. Kabov, Calculation 
of the heat flux near the liquid-gas-solid contact line, Applied Mathematical Modelling 40 (2) (2016) 1029-1037.

[33] J. N. Israelachvili, Intermolecular and surface forces, Academic press, 1992.

[34] Z. Pan, S. Dash, J. A. Weibel, S. V. Garimella, Assessment of water droplet evaporation mechanisms on hydrophobic and superhydrophobic substrates, Langmuir 29 (51) (2013) 1583115841.

[35] I. Malavasi, B. Bourdon, P. Di Marco, J. De Coninck, M. Marengo, Appearance of a low superheat "quasi-leidenfrost" regime for boiling on superhydrophobic surfaces, International Communications in Heat and Mass Transfer 63 (2015) 1-7.

[36] J. Drelich, E. Chibowski, Superhydrophilic and superwetting surfaces: definition and mechanisms of control, Langmuir 26 (24) (2010) 18621-18623.

[37] O. Raghu, J. Philip, Thermal properties of paint coatings on different backings using a scanning photo acoustic technique, Measurement Science and Technology 17 (11) (2006) 2945.

[38] T. L. Bergman, F. P. Incropera, A. S. Lavine, Fundamentals of heat and mass transfer, Wiley, 2011.

[39] S. Siedel, S. Cioulachtjian, A. J. Robinson, J. Bonjour, Integral momentum balance on a growing bubble, Physics of Fluids 25 (12) (2013) 123301.

[40] L. Kirkup, R. B. Frenkel, An Introduction to Uncertainty in Measurement: Using the GUM (Guide to the Expression of Uncertainty in Measurement), Cambridge University Press, 2006.

[41] M. Gibbons, C. Howe, P. Di Marco, A. Robinson, Local heat transfer to an evaporating sessile droplet in an electric field, in: Journal of Physics: Conference Series, Vol. 745, IOP Publishing, 2016, p. 032066.

[42] S. Dash, S. V. Garimella, Droplet evaporation dynamics on a superhydrophobic surface with negligible hysteresis, Langmuir.

[43] E. F. Crafton, W. Z. Black, Heat transfer and evaporation rates of small liquid droplets on heated horizontal surfaces, International Journal of Heat and Mass Transfer 47 (6) (2004) 11871200.

[44] E. Y. Gatapova, A. A. Semenov, D. V. Zaitsev, O. A. Kabov, Evaporation of a sessile water drop on a heated surface with controlled wettability, Colloids and Surfaces A: Physicochemical and Engineering Aspects 441 (2014) 776-785.

[45] B. Horacek, K. Kiger, J. Kim, Single nozzle spray cooling heat transfer mechanisms, International Journal of Heat and Mass Transfer 48 (8) (2005) 1425-1438.

[46] C. Sodtke, P. Stephan, Spray cooling on micro structured surfaces, International Journal of Heat and Mass Transfer 50 (19) (2007) 4089-4097. 